\title{
Does Quantum Electrodynamics Have an Arrow of Time? ${ }^{1}$
}

\author{
David Atkinson \\ University of Groningen, The Netherlands
}

\begin{abstract}
Quantum electrodynamics is a time-symmetric theory that is part of the electroweak interaction, which is invariant under a generalized form of this symmetry, the PCT transformation. The thesis is defended that the arrow of time in electrodynamics is a consequence of the assumption of an initial state of high order, together with the quantum version of the equiprobability postulater.atkinson@rug.nl
\end{abstract}

Keywords: time, arrow, quantum electrodynamics.

\section{Introduction}

When the Maxwell equations are used in classical electrodynamics, it is commonly assumed that the only solutions of physical relevance are those that are obtained by employing the retarded Green's function, without the addition of a component of the electromagnetic field that is free from sources and sinks. This Sommerfeld condition seems at first sight to implement the

\footnotetext{
${ }^{1}$ The gestation period of this paper began when I was visiting The Centre for Time at Sydney University in 2002, and I am particularly indebted to Huw Price and to Mathias Frisch, with whom I discussed the problem of time's arrow in electromagnetic radiation. The original plan for all three of us to collaborate in writing one paper came to naught, due to disagreements about how to solve that problem; but we did manage to agree to write three separate papers, a decision that received further stimulus when we were all three invited by Michel Ghins to take part in a workshop, 'The Arrow of Time: Physics and Philosophy', in Louvain-la-Neuve in 2004. I am in agreement with Price, against Frisch, that the genesis of the radiation arrow is to be sought in a temporal asymmetry of sources, a fact-like initial condition. On the other hand, I think that a purely classical treatment, of the kind that Price gives, runs into trouble on close scrutiny, because a consistent classical energy distribution function does not exist for the electromagnetic field, and so the statistical arguments concerning entropy increase cannot get off the ground.

I would also like to thank Huw Price for financial and other support at the Centre for Time, and Michel Ghins in Louvain, as well as Dennis Dieks and Jos Uffink in Utrecht, who first suggested the possibility of publishing our three articles together.
} 
everyday expectation that a cause should temporally precede its effect, and hence to forge an electromagnetic arrow of time, pointing by fiat from the past to the future.

However, as Huw Price (2005) eloquently argues, the Sommerfeld condition is neither a necessary, nor a sufficient condition for a solution of the Maxwell equations to be asymmetric in time. Yet we do indeed observe the frequent occurrence of expanding light fronts, whether from lamps or from stars, and never their time-reversed twins. What is the reason for this observed temporal asymmetry? Does it have its origin perhaps outside the framework of electrodynamics? Are we seeing the effect of a master arrow, the one which ordains that water waves on a pond are always observed to expand from a stone that is dropped in the middle, and never to contract coherently to that middle point, arriving just as the stone hits the water? In this paper we answer the last two questions positively by considering the quantum theory of electrodynamics. This should be seen as the completion and ultimate justification of purely classical attempts, based on Maxwell's electromagnetic theory, to locate the source of the time-asymmetry of the observed solutions of the equations in the boundary conditions, rather than in the equations themselves.

Quantum electrodynamics (QED) is time-symmetric; and the scattering of photons and electrons bears a superficial resemblance to molecular collisions in the theory of gas kinetics. In both cases, each elementary collision process is fully reversible; and not only is the temporal inverse of each process another process that is permitted by the dynamics, but the rates involved in multiple processes are the same in either direction of time. The origin of an electromagnetic arrow is thus analogous to that of the statistical mechanical arrow in the theory of molecular collisions.

In the classical statistical mechanical treatment of molecular collisions, one must add the 'equiprobability postulate' to the Newtonian equations of mechanics. This postulate states that equal hypervolumes on constantenergy manifolds of phase space are to be assigned the same probability of occupation, and it thereby defines what is called the 'natural measure'. The idea is made the more attractive by the fact that the natural measure is invariant under any canonical transformation of the coordinates. Moreover, Liouville's theorem guarantees that the hypervolume of a constant-energy region of phase space remains invariant in time, so that the probability of its occupation likewise does not depend on the time, so long as one uses the natural measure. These attractive properties of the natural measure have misled 
some writers into regarding the equiprobability postulate as part and parcel of Newtonian mechanics. However, any independent, continuous functions of the positions and momenta of the molecules could be used as alternative variables, and in general a uniform probability density on the original variables does not imply uniformity on the new ones. The fact that uniformity with respect to the Cartesian positions and momenta of the molecules survives canonical transformation is no justification for assuming uniformity in the first place ${ }^{2}$. The best that can be said is that the assumption of the natural measure is a postulate that has to be added to the standard corpus of Newtonian mechanics in order to yield classical statistical mechanics, and that the latter enjoys much empirical success. In this way the equiprobability postulate receives a posteriori confirmation, even if its apparent independence from Newton's mechanics remains a thorn in the theoretician's side.

The important point is that the natural measure does not favour one direction in time above the other: it cannot in itself give rise to the temporal asymmetries that are so evident in the world. These asymmetries are traced back to a condition of high order at a particular time, an epoch that is in what we call the remote past. The equiprobability postulate then makes it overwhelmingly probable that order will decrease toward later times. Technically, one conditionalizes by using a low-entropy boundary condition at one extremity of a finite period of time, without any parallel conditionalization at the other temporal extremity. The first extremity we call the remote past, the second the future.

This account is the conclusion of a long series of attempts to come to grips with the thermodynamical arrow of time, starting with the seminal work of Boltzmann. It applies to the classical statistical mechanics of, for example, colliding molecules of gas. When the same treatment was extended to radiation, the latter being treated as an ensemble of harmonic oscillators, it was found that Boltzmann's recipe for the distribution of energy over the frequencies does not work. It leads to the Rayleigh-Jeans distribution, with the absurd result that the total energy of any sample of radiation is infinite (the UV catastrophe).

We reach here the end of the classical road and must needs take the quan-

\footnotetext{
${ }^{2}$ The ergodic hypothesis was meant to provide a rationale for the equiprobability postulate, but since it cannot be demonstrated to be true for interesting systems, it is indeed merely a postulate, on a par with the equiprobability postulate itself. Moreover, the passage from ergodicity to equiprobability is fraught with technical difficulty and uncertainty, so it seems preferable to take the equiprobability postulate as the primitive notion.
} 
tum highway. Max Planck avoided the UV catastrophe by the hypothesis of a fundamental quantum of action, leading to the Planck distribution of energies instead of the disastrous Rayleigh-Jeans distribution. In the quantum theory of electromagnetic radiation, the equations are invariant under reversal of time, and there is a quantum version of the equiprobability postulate. However, the statistics, i.e. the method of counting distinct microscopic configurations, is not the same in quantum as in classical physics. Boltzmann counting is replaced by Bose-Einstein counting, and this immediately yields the Planck distribution, which we may see as a consequence of the assumption of equiprobability, quantum style. In QED, a somewhat better case can be made for the adoption of the equiprobability postulate than is possible in the classical theory. It is supported by general arguments concerning the number and nature of the conserved, additive quantities in a relativistic quantum field theory like QED; but it remains finally as an extra assumption that is justified a posteriori by the undoubted success of quantum statistical mechanics.

In Sect. 2, the meaning of the T-symmetry of quantum electrodynamics is spelled out in detail, and its breakdown in the electroweak extension of that theory is explained. Violations of the $\mathrm{P}, \mathrm{C}$ and $\mathrm{T}$ symmetries are illustrated in Sect. 3, and the PCT theorem is also elucidated. In Sect. 4, statistical mechanics in a quantum setting is further discussed, a complication being that various competing interpretations of the quantum mechanical measurement process have to be assessed.

\section{Quantum Electrodynamics}

It is sometimes claimed that pure emission of light, without previous absorption, is possible, and even common, whilst pure absorption, without subsequent residual emission, is impossible, or at least very unusual. In any case, so it is argued, here is a clear case of temporal asymmetry, here is a putative electromagnetic arrow of time.

The most advanced and empirically successful theory of light that we have to date is quantum electrodynamics. Compton scattering is the name given to the scattering of particles of light (photons) by particles of electricity (electrons), and the basic process is pictured in the Feynman diagram shown 
in Fig. 1:

Figure 1

Compton scattering

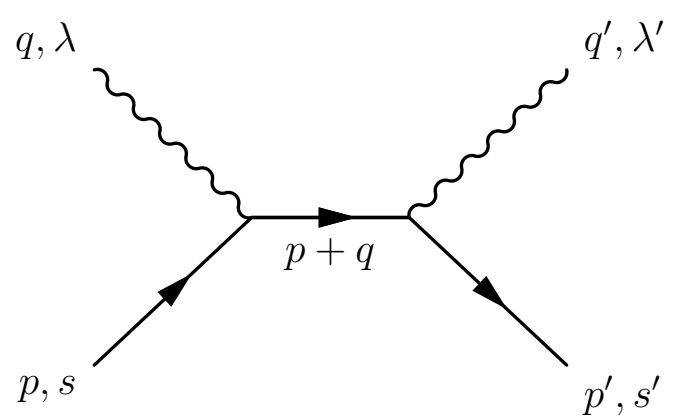

An electron of momentum $p$ and spin $s$ absorbs a photon of momentum $q$ and polarization $\lambda$. The intermediate electron has momentum $p+q$, since momentum is conserved at each vertex, and the final state consists of an electron of momentum $p^{\prime}$ and spin $s^{\prime}$, and a photon of momentum $q^{\prime}$ and polarization $\lambda^{\prime}$.

Absorption of light without re-emission corresponds in this elementary Feynman diagram to the case $q^{\prime}=0$, for if the momentum of the outgoing photon is zero, its energy is likewise zero; in other words, there is no outgoing radiant energy, i.e. no outgoing radiation. However, that is kinematically impossible if the electron is in a free state, before and after absorption of the initial photon, because of the conservation of energy and momentum, and the so-called mass-shell condition. The mass-shell condition is the relativistic requirement that the square of the energy of a particle, minus the square of its momentum, must be equal to the square of the mass of the particle (in units such that the speed of light in vacuo is unity). The mass-shell condition applies to the initial and final particles, but not to any intermediate, 'virtual' particles (like the electron of momentum $p+q$ in Fig. 1). At first sight, this would appear to corroborate the claim that absorption of light, without residual re-emission, is impossible, and to support the claim that there is an inbuilt arrow of time.

However, precisely the same reasoning also precludes the Compton process in which the momentum of the initial photon is zero, $q=0$, rather than that of the final photon. Thus it would seem that pure emission, like pure absorption, is impossible for a free electron, and so there is no temporal asymmetry here after all.

Although pure emission of light does not take place from a free electron, it is another matter for an electron that is in a bound state, for example 
in an atom. If the atom is not in its ground state, but rather in one of its energetically excited states, it can undergo a transition to the ground state, with the emission of a photon. The modified Feynman diagram is as shown in Fig. 2:

Figure 2

Pure emission

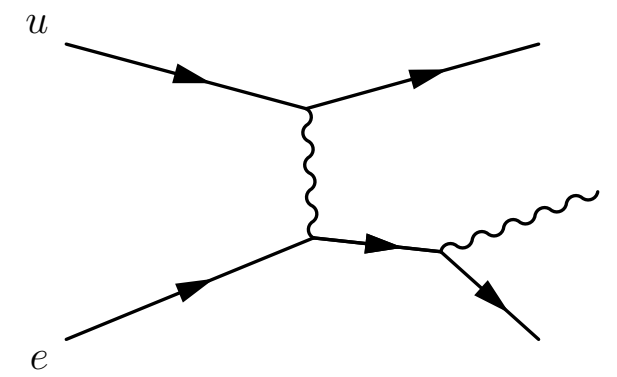

Instead of an incoming, real photon, there is now a virtual photon which accounts for the interaction that binds the electron $(e)$ to, for example, an up quark $(u)$ in a proton in the nucleus of the atom. The energy of the emitted photon is equal to the energy of excitation that the atom originally had.

Pure emission of light, without prior absorption of a photon, is thus possible after all, on condition that the electron is bound. Does the inverse process exist? Indeed it does, and it corresponds, in the case already considered, to the absorption of a photon by an atom, in its ground state, resulting in an excited atom. The process is pictured in Fig. 3, the time reverse of Fig. 2:

Figure 3

Pure absorption

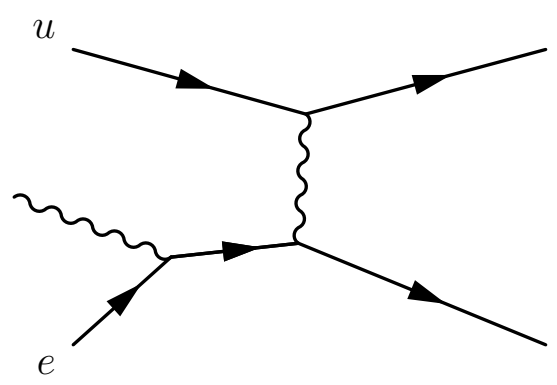

Although the absorption of a photon by an atom is possible, this can be a pure process, with no re-emission, only if the photon's energy is very finely tuned to be equal to the excitation energy of one of the excited states of the atom. This is more difficult to arrange than the inverse process, as pictured in Fig. 2. Indeed, often the only practical way to produce photons of the 
right frequency is through the preliminary de-excitation of other atoms that are already in the excited state or states in question.

At the level of the individual Compton process, although a given transition is not in general time-symmetric, since the initial and final momenta of the photon and the electron are usually different, nevertheless for each transition there is another one which is related to the first by time inversion. The most extreme example is a pure absorption, which is the time-inverse of a pure emission. The time-inversion invariance of QED guarantees this Tsymmetry. The set of all possible Compton scatterings is symmetric in time: no arrow of time is defined by the phenomena of emission and absorption of photons $^{3}$.

It should be explained that the Feynman diagram of Fig. 1 is not the only contribution to Compton scattering at the two-vertex level. To the amplitude corresponding to that Feynman diagram, one needs to add the amplitude of the crossed diagram, shown in Fig. 4 (in which the spin and polarization indices have been suppressed). Whereas the Feynman diagram of Fig. 1 can be interpreted as the absorption of an incoming photon by the electron, and the subsequent emission of an outgoing photon (generally of a different energy), the Feynman diagram of Fig. 4 suggests the retrocausal scenario in which the emission of an

Figure 4

Crossed diagram

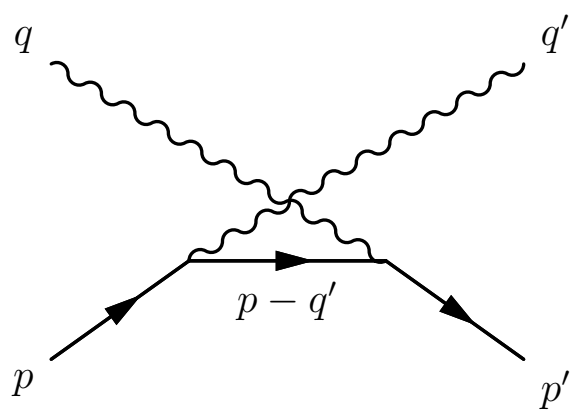

outgoing photon takes place before the absorption of the incoming photon, which in some sense causes the emission, although this has already taken place. It is only the sum of the contributions of Figs. 1 and 4 that is T-symmetric. The same thing happens at all perturbative levels in QED, a consequence of the fact that the theory is time-reversal invariant. The

\footnotetext{
${ }^{3}$ The electromagnetic fields themselves are not T-invariant, for the magnetic induction changes its sign under time-reversal, while the electric field does not. The T-invariance of QED refers to scattering processes alone.
} 
Green's function that is used to calculate scattering amplitudes can be written as the sum of three parts (see Atkinson, 2000, p. 48): a retarded Green's function, an advanced Green's function, each with the same strength, and a self-interaction term, reflecting the fact that an electron interacts with the electromagnetic field that it produces itself.

QED is a T-symmetric field theory, and it describes the interaction of photons and electrons correctly to very high accuracy, but it is not the ultimate theory relating to these particles. Electrons participate also in the so-called weak interaction, which is not exactly symmetric under time reversal: there are some weak effects that violate T-symmetry by about one part in a thousand. All three generations are needed to generate these effects (up and down quarks in the first, charmed and strange quarks in the second, and top and bottom quarks in the third generations). Since the quarks carry electric charge, they couple to photons, and hence they contribute to Compton scattering in higher orders. In Fig. 5 a T-violating contribution to the Compton process is shown. The quark loop, involving the up, strange and top quarks, coupled to the electron line by charged weak gauge bosons, $W$, gives rise to violation of T-symmetry. There are many more diagrams like this, which are not T-invariant in the electroweak theory (as the unification of QED with the weak interaction is called). Fig. 5 is just one example, and the T-violating contributions of the various diagrams do not cancel one another: there is a net, if tiny, violation of T-symmetry in QED, due to the weak effects.

Figure 5

T-violation

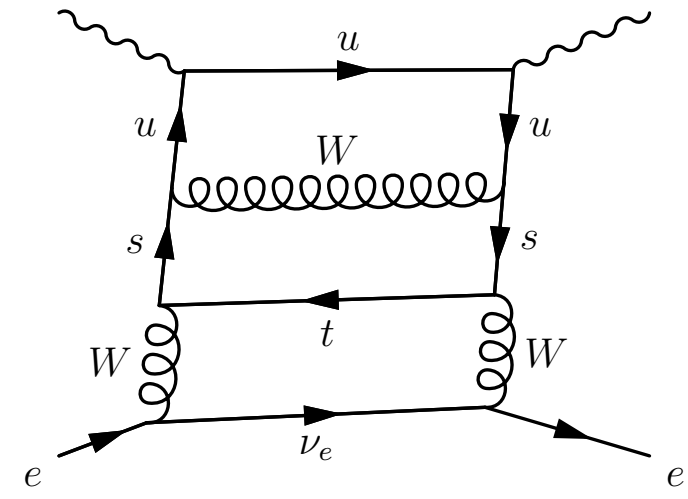

Usually this small violation of time-reversal symmetry is neglected in discussions of the radiative arrow of time, on the grounds that it is so small that it could not explain the grossly asymmetric effects that are experienced in 
electromagnetism. These effects are often handled at the classical level by adjoining to the Maxwell equations the Sommerfeld condition, which amounts to the recipe to use the retarded, and not the advanced field solutions of these equations. It does not seem, indeed, that such a recipe could ever be a consequence of the lack of T-invariance due to weak effects. Nevertheless, one might well make a point of principle: because of the breaking of T-symmetry by the weak interaction, it is not true that there is no microscopic arrow of time in electrodynamics. Whether or not there is more to be said concerning the origin of a macroscopic arrow, the mere whiff of directionality at the level of the fundamental laws calls into question the claim that the distinction between past and future is merely one of our standpoint as agents (Price, 1996, p. 168).

The weight of the criticism is more apparent than real, for the electroweak theory, like all local quantum field theories, possesses a symmetry called PCT-invariance, which is a natural generalization of T-invariance. To explain what conservation of PCT involves, I first introduce the $\mathrm{P}$ and $\mathrm{C}$ operations, and then give some examples in which $\mathrm{P}, \mathrm{C}$ and $\mathrm{T}$ are separately violated.

\section{Violation of $\mathrm{P}, \mathrm{C}$ and $\mathrm{T}$ Symmetries}

The violation of $\mathrm{P}$ symmetry was first demonstrated by Chieng-Shiung $\mathrm{Wu}$ (1957) by studying the decay of the radioactive cobalt-60 nucleus, following a suggestion of Lee and Yang (1956).

In Fig. 6, the sphere at the left depicts a nucleus of $\mathrm{Co}^{60}$, which has a spin, indicated as a classical direction of rotation for convenience. The cobalt nucleus decays through the weak interaction into a nucleus of nickel, producing an electron, which is detected, and an antineutrino, which escapes detection:

$$
\mathrm{Co}^{60} \longrightarrow \mathrm{Ni}^{60}+e^{-}+\bar{\nu}_{e}
$$

Only one electron is produced per decay, and sometimes this escapes 'upwards' (with respect to the nuclear spin), and sometimes 'downwards'. The picture at the left of Fig. 6 should be regarded as a composite of many nuclei; and Mrs Wu found experimentally that the electron more often went downwards than upwards, as suggested by the picture.

The parity operation, denoted $\mathrm{P}$, prescribes the inversion of all spatial coordinates, or equivalently reflection in a mirror. The picture in the middle of Fig. 6 is obtained by imagining a vertical mirror to the right of the 
leftmost nucleus, producing an image in which the direction of spin has been reversed, since reflection interchanges left and right. The picture on the extreme right is this same mirror image, rotated through 180 degrees, so that it can more readily be compared with the picture on the left. Evidently there is a difference between the pictures on the left and the right. In our world, the one we share with $\mathrm{Mrs} \mathrm{Wu}$, the probability of emission of the decay electron is greater in the direction opposed to the nuclear spin than in the direction of the spin, whereas in the mirror world the matter is the other

\section{P-violation}

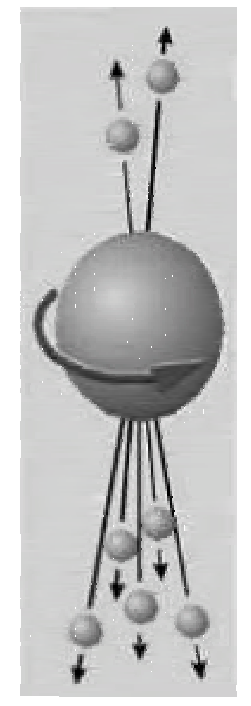

Our world
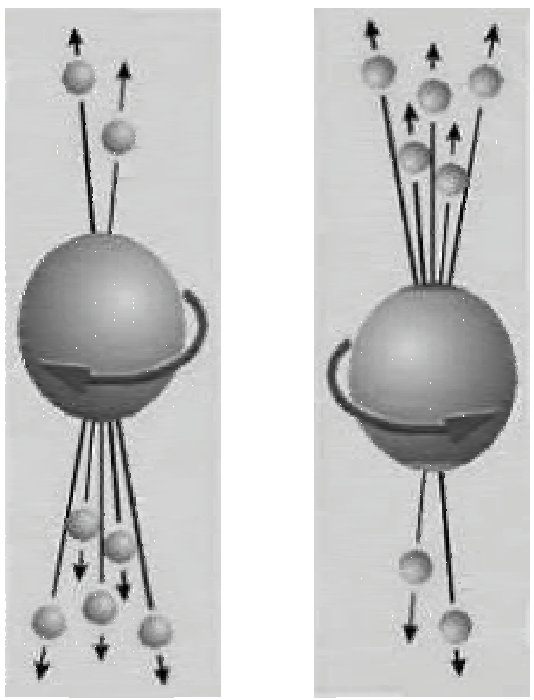

Mirror world

Figure 6

way about. If $\mathrm{P}$ were a good symmetry - we say if $\mathrm{P}$ were conserved - the probabilities in either direction would be the same, so that the composite of many decays would be indistinguishable from its mirror image. This is not the case, for there is a statistical difference between the situation shown at the left and that at the right. Thus $\mathrm{P}$ is not a good symmetry in our world, at any rate as far as reactions involving the weak interactions are concerned.

The charge conjugation operator, $\mathrm{C}$, replaces all particles by their antipar- 
ticles, without affecting the spatial characteristics in any way. In particular, no reflection is involved, only a transformation of the nature of the particles themselves. Thus the cobalt nucleus, pictured again in Fig. 7, but now in the middle, is transformed into an anticobalt nucleus, made up of antiprotons and antineutrons, and the electrons are transformed into positrons. This transformation has been depicted by replacing the image by its negative, as can be seen on the right of Fig. 7 .

\section{C-violation}

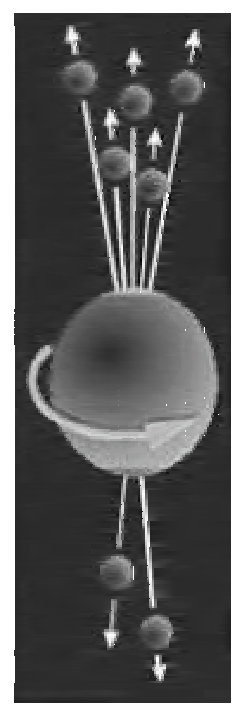

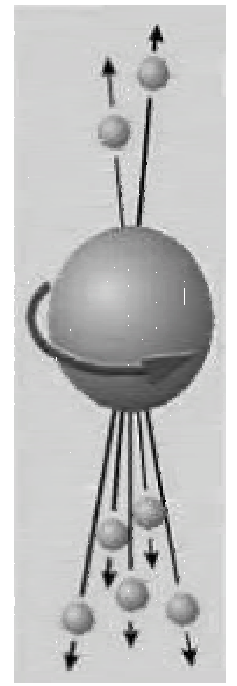

Our world

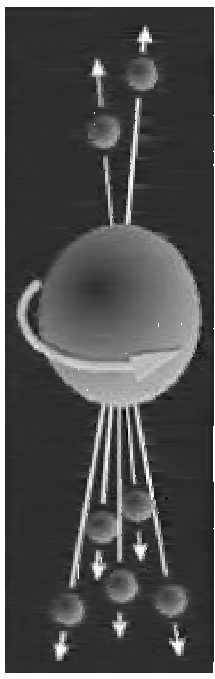

C-transform world

Figure 7

However, this negative picture does not correctly represent how a nucleus of anticobalt would decay in our world. Although present technology does not permit the actual construction of a piece of anticobalt, theory predicts that if Mrs $\mathrm{Wu}$ had rerun her experiment with such a sample of antimatter, she would have observed more positrons leaving the nucleus along the direction of the spin, rather than opposed to it, as shown at the left of Fig. 7. The picture at the right is the C-transform of the decay of cobalt-60, whereas the picture at the left indicates the way that anticobalt-60 would in fact decay. The mismatch between these pictures illustrates the violation of charge conjugation symmetry. 
Although the decay of cobalt-60 exhibits breakdown of $\mathrm{P}$ and $\mathrm{C}$ symmetry, it does respect PC conservation. That is, if we reflect the system in a mirror, and change particles into antiparticles as well, then the decay in the imaginary $\mathrm{PC}$ world is indistinguishable from that in our world, as indicated in Fig. 8. The two pictures on the left show respectively the way anticobalt-60 and cobalt-60 decay in our world, and are just as in Fig. 7.

\section{PC-conservation}

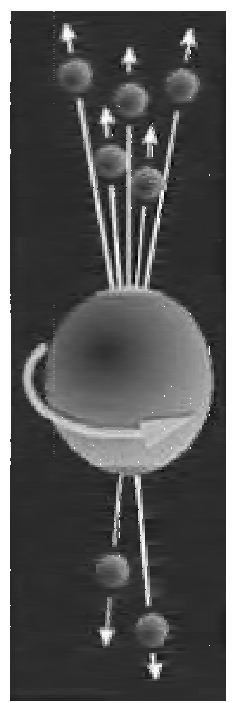

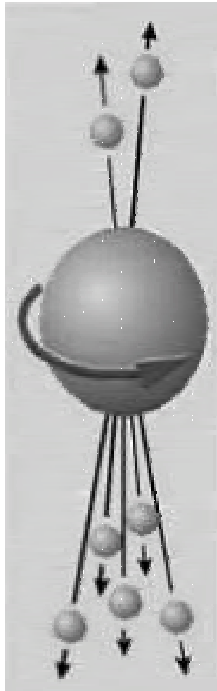

Our world
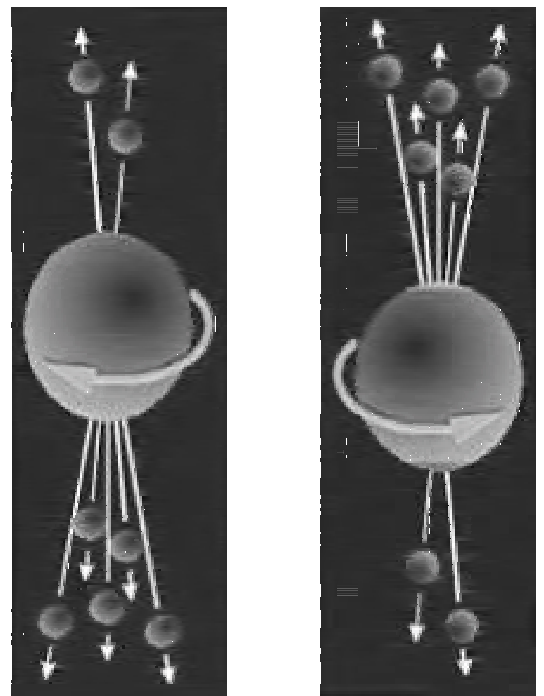

PC-transform world

Figure 8

The third picture from the left is the PC transform of the cobalt-60 decay to its immediate left, in which the spin direction of the nucleus has been reversed by $\mathrm{P}$, and all particles have been turned into antiparticles by $\mathrm{C}$. Finally, the picture on the extreme right is simply this third picture, rotated through 180 degrees. Evidently the pictures on the extreme left and right are identical, indicating that $\mathrm{PC}$ is here a good symmetry.

In the decay of the neutral kaon, $K^{0}$, on the other hand, PC symmetry is also violated - and not simply $\mathrm{P}$ and $\mathrm{C}$ separately, as in the case of $\mathrm{Co}^{60}$. The quantum field that describes a kaon, $\phi\left(K^{0}\right)$, is pseudoscalar, i.e. its sign is changed by the parity operator, $\mathrm{P} \phi\left(K^{0}\right)=-\phi\left(K^{0}\right)$, and similarly for the 
antikaon quantum field, $\mathrm{P} \phi\left(\bar{K}^{0}\right)=-\phi\left(\bar{K}^{0}\right)$. The $\mathrm{C}$ operator interchanges a kaon and an antikaon field, $\mathrm{C} \phi\left(K^{0}\right)=\phi\left(\bar{K}^{0}\right)$ and $\mathrm{C} \phi\left(\bar{K}^{0}\right)=\phi\left(K^{0}\right)^{4}$. Thus the operation $\mathrm{PC}$ interchanges particle and antiparticle, and introduces a minus sign: $\mathrm{PC} \phi\left(K^{0}\right)=-\phi\left(\bar{K}^{0}\right)$ and $\mathrm{PC} \phi\left(\bar{K}^{0}\right)=-\phi\left(K^{0}\right)$. It follows that the combination $\phi\left(K_{S}^{0}\right)=\phi\left(K^{0}\right)-\phi\left(\bar{K}^{0}\right)$ is even under PC, that is, $\operatorname{PC} \phi\left(K_{S}^{0}\right)=\phi\left(K_{S}^{0}\right)$.

This combination of fields, called $K$-short, decays with a relatively short lifetime into two pions,

$$
K_{S}^{0} \longrightarrow \pi^{+}+\pi^{-} .
$$

On the other hand, the odd combination, $\phi\left(K_{L}^{0}\right)=\phi\left(K^{0}\right)+\phi\left(\bar{K}^{0}\right)$, called $K$ long, for which $\mathrm{PC} \phi\left(K_{L}^{0}\right)=-\phi\left(K_{L}^{0}\right)$, decays preferentially into three pions,

$$
K_{L}^{0} \longrightarrow \pi^{+}+\pi^{-}+\pi^{0}
$$

and this occurs with a relatively long lifetime. The reason for the differing decay products is that a two-pion state is even under PC, whereas a threepion state is odd. The exhibited decays of $K_{S}^{0}$ and $K_{L}^{0}$ therefore conserve PC, i.e. they are invariant under the combined operations of parity and charge conjugation. If this were the end of the story, the system would involve violation of $\mathrm{P}$ and $\mathrm{C}$, but conservation of $\mathrm{PC}$ symmetry, as in the decay of cobalt-60. However, there is more. Although $K_{L}^{0}$ indeed decays preferentially into three pions, it is found experimentally that there is a small branching ratio into two pions:

$$
K_{L}^{0} \longrightarrow \pi^{+}+\pi^{-},
$$

which would be strictly forbidden if PC symmetry were conserved. The observed ratio (see Hagiwara, 2002) of the amplitudes for the PC-forbidden and the PC-allowed decays is a couple of parts per thousand:

$$
\left|\frac{\text { Amplitude }\left(K_{L}^{0} \rightarrow \pi^{+}+\pi^{-}\right)}{\operatorname{Amplitude}\left(K_{L}^{0} \rightarrow \pi^{+}+\pi^{-}+\pi^{0}\right)}\right|=(2.29 \pm 0.02) \times 10^{-3} .
$$

This violation of PC symmetry was first discovered by Cronin and Fitch (1964), and it is an indirect indication of the violation of T symmetry, as I shall explain in a moment. However in 1998 a direct violation of T symmetry was measured for the first time, also in the kaon system, and I first turn to this matter.

\footnotetext{
${ }^{4}$ More generally, a phase factor may be introduced, but for simplicity of notation, I choose this to be unity.
} 
A kaon can turn into an antikaon, as shown in this Feynman diagram:

Figure 9

$K^{0} \bar{K}^{0}$ transition

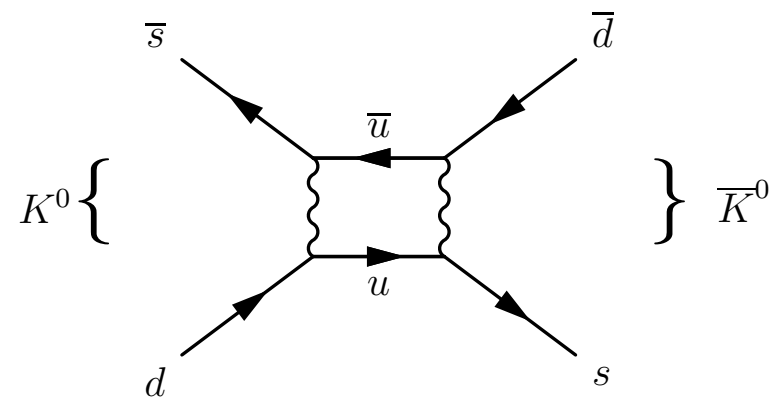

As part of the CPLEAR experiment at CERN (Angelopoulos, 1998), it was determined that the speed of the transition $\bar{K}^{0} \longrightarrow K^{0}$ is half a percent greater than that of the transition $K^{0} \longrightarrow \bar{K}^{0}$ :

$$
\frac{\operatorname{Rate}\left(\bar{K}^{0} \longrightarrow K^{0}\right)-\operatorname{Rate}\left(K^{0} \longrightarrow \bar{K}^{0}\right)}{\operatorname{Rate}\left(\bar{K}^{0} \longrightarrow K^{0}\right)+\operatorname{Rate}\left(K^{0} \longrightarrow \bar{K}^{0}\right)}=(6.6 \pm 2.3) \times 10^{-3} .
$$

This experiment was a tour de force, involving the Low Energy Antiproton Ring at CERN, and sophisticated online analysis of the decay products of proton-antiproton collisions. One possible product of the collision is $p \bar{p} \longrightarrow \pi^{-} K^{+} \bar{K}^{0}$, and this intermediate state can be picked out by deflecting the $K^{+}$kaon in a magnetic field and subsequently identifying it. The accompanying $\bar{K}^{0}$, which is not deflected, progresses typically several metres before decaying. It may decay into two or three pions, but it may also decay as follows: $\bar{K}^{0} \longrightarrow \pi^{+} e^{-} \bar{\nu}_{e}$. The corresponding decay of a $K^{0}$ kaon is $K^{0} \longrightarrow \pi^{-} e^{+} \nu_{e}$. Hence if a $\bar{K}^{0}$ has been isolated, and it decays into $\pi^{-} e^{+} \nu_{e}$ rather than $\pi^{+} e^{-} \bar{\nu}_{e}$, it must be that the $\bar{K}^{0}$ has transformed into a $K^{0}$ before decay. In this way the rate of the transformation $\bar{K}^{0} \longrightarrow K^{0}$ can be assessed. The rate for the inverse reaction, $K^{0} \longrightarrow \bar{K}^{0}$, is measured by looking at the decay product $p \bar{p} \longrightarrow \pi^{+} K^{-} K^{0}$ in a similar way, isolating the $K^{0}$ and measuring the rate of production of $\pi^{+} e^{-} \bar{\nu}_{e}$ rather than $\pi^{-} e^{+} \nu_{e}$, this being indicative of the intermediate transformation $K^{0} \longrightarrow \bar{K}^{0}$.

In this way a breakdown of $\mathrm{T}$ symmetry has been demonstrated, independently of the PC violation that had already been established by Cronin and Fitch in 1964. However, the violation of T and of PC are not independent of one another, because of the PCT theorem (Pauli, 1955), which states that PCT symmetry is a property of all local, relativistically covariant field 
theories. Hence the PC violation that Cronin and Fitch measured must be compensated by a $\mathrm{T}$ violation of opposite sign, so that the violations of $\mathrm{PC}$ and of $\mathrm{T}$ symmetry cancel one another.

Although the PCT theorem is very general, it is after all a theoretical result, and as such it rests upon assumptions that may be questioned, in particular the claim that nature may be accurately described by a local quantum field theory. This assumption of locality involves the interaction of quantum fields at the same space-time point, which leads to problematic ultraviolet divergences, that are removed by the controversial infinite renormalization scheme. Hence the experimental testing of PCT invariance is important, and this has been performed in many ways (see Hagiwara, 2002). PCT symmetry implies in particular that the mass of every particle must be equal to the mass of the corresponding antiparticle, and the most accurate result as of 2002, once more in the $K^{0} \bar{K}^{0}$ system, is

$$
\left|\frac{\operatorname{mass}\left(\bar{K}^{0}\right)-\operatorname{mass}\left(K^{0}\right)}{\operatorname{mass}\left(K^{0}\right)}\right|<10^{-18},
$$

which indeed is a satisfyingly tiny upper bound on a difference that is predicted to be zero.

\section{Quantum Statistical Mechanics}

There is no arrow of time to be found in the structure of QED; and more generally the electroweak theory, in which QED is embedded, is invariant under the PCT transformation. The theory of the scattering of photons by electrons, or indeed by any charged particles, is symmetric under inversion of the direction of time, on condition that one reflects the space coordinates, and interchanges particles and antiparticles as well. In the following considerations, we shall refer to T-invariance for short, which applies to pure QED; but everything should be understood more properly in the generalized sense of PCT-invariance.

The fundamental arrow of time is defined by the time-sense from an early, highly-ordered state of the universe, to a later, less ordered state. There is detailed parallelism between the quantum and the classical versions of this account. In both cases, one needs two assumptions: 
a. Equiprobability postulate: equal hypervolumes on a constant-energy manifold of phase space are assigned the same probability. This leads to the natural measure.

b. Past postulate: there is high order at one temporal extremity; i.e. low probability at one end of time, calculated with the natural measure.

In quantum theory, the equiprobability postulate is theoretically attractive for the same reason as it is in classical theory: it is invariant under (quantum) canonical transformation. The probability associated with a given hypervolume remains constant in time, thanks to the quantum version of Liouville's theorem. The crucial distinction between quantum and classical statistics lies in the manner of counting microstates. The theoretical derivation of equiprobability from the ergodic hypothesis is at least as problematical in quantum as in classical theory, so we prefer to take equiprobability as the basic postulate. A justification for the assumption of equiprobability is provided by the wealth of successful predictions that have been made on the basis of quantum statistics.

Whereas the reason for believing the correctness of the equiprobability postulate is built up from many pieces of empirical evidence, the reason for believing the past postulate is based mainly on one observational fact. The relic radiation from the early universe (NASA, 2004) has an extremely high degree of isotropy. It is thereby established that the ordering of matter and radiation shortly after the Big Bang was high, as estimated by the natural measure.

This might seem to be the end of the story: the interaction of radiation and matter is time symmetric, and the observed asymmetries in time are consequences of the two postulates that were given above, the first one being time-symmetric, the second one breaking the time symmetry by postulating a high degree of order at one temporal extremity, but not at the other.

However, QED is a quantum theory, and we shall now address briefly the problem of measurement in quantum theory, or more generally the status of the notorious collapse of the wave function. Might this not be the source of a further time asymmetry? According to Copenhagen orthodoxy, the unitary evolution in time of a state vector is reversible, but the collapse engendered by an 'observation' is irreversible. Many people have been dissatisfied with this account, and some of the 'solutions' to the observation problem are as follows: 
1. The many-worlds interpretation of Everett (1957).

2. The transactional interpretation Cramer (1986).

3. Decoherentism (see Zeh, 2001).

4. GRW physical collapse (Ghirardi, Rimini and Weber, 1986).

The many-worlds interpretation may have an arrow of time, depending on the precise details; but the transactional interpretation does not, or at least it can be cast into a time-symmetric form, in which the 'offer' and 'acceptance' waves are simply interchanged by the T-transformation. Decoherentism, in which phase correlations remain, but become less and less relevant, is not explicitly time-asymmetric.

The GRW physical collapse mechanism has been championed recently by Albert (2000), who claims that the postulated spontaneous destruction of quantum correlations provides in one fell swoop both the underlying quantum probability measure and a rationale for the equiprobability thesis, which therefore no longer has the status of an independent assumption. If Albert is right, one would be able to dispense with one of the two postulates, at the expense, it is true, of the GRW hypothesis itself. An objection to this way of grounding the arrow of time is that it offends a philosophical sensibility, in that there would seem to be two time arrows: the first is the supposed temporal sense in which the GRW mechanism operates, and the second is the postulate that there is high order at one temporal extremity of the world. The latter postulate does not follow from the GRW hypothesis, and so it seems that Albert's solution amounts simply to replacing the equiprobability postulate by the GRW postulate. He might have a better bargain, as it were, because he buys the quantum probability measure at no extra cost; but the fact that the posited GRW arrow of time is not in itself sufficient to account for the observed temporal asymmetries is a source of embarrassment. The objection is of course not decisive, for the GRW proposal must stand or fall on the basis of experiment. Despite nearly twenty years of effort, there is no evidence that the model is correct.

\section{References}

Albert, D. Z. (2000). Time and chance. Cambridge: Harvard University Press. 
Angelopoulos, A. et al. (1998). First direct observation of time-reversal non-invariance in the neutral kaon system. Physics Letters B 444, 43-51.

Atkinson, D. (2000). Quantum mechanics and retrocausality. In N. Dadhich and A. Kembhavi (eds.), The universe, visions and perspectives (35-50). Dordrecht: Kluwer Academic Publishers.

Cramer, J.G. (1986). The transactional interpretation of quantum mechanics. Reviews of Modern Physics 58, 647-688.

Christenson, J. H., Cronin, J.W., Fitch, V.L and Turlay, R. (1964). Evidence for the $2 \pi$ decay of the $K_{20}$ meson. Physical Review Letters 13, 138-140.

Everett, H. (1957) 454-462. 'Relative state' formulation of quantum mechanics. Reviews of Modern Physics 29, 454-462.

Ghirardi, G.C., Rimini, A. and Weber, T. (1986). Unified dynamics for microscopic and macroscopic systems. The Physical Review D 34, 470-491.

Hagiwara, K. et al., the particle data group. (2002). The Physical Review D66. and http://pdg.lbl.gov/

Lee, T.D. and Yang, C.N. (1956). Question of parity conservation in weak interactions. The Physical Review 104, 254-258.

NASA (2004). Legacy archive for microwave background analysis. http://lambda.gsfc.nasa.gov/product/

Pauli, W. (1955). Exclusion principle, Lorentz group and reflection of spacetime and charge. In W. Pauli, L. Rosenfeld V. Weisskopf (eds.), N. Bohr and the development of physics. London: Pergamon Press.

Price, H. (1996). Time's arrow and Archimedes' point. Oxford: Oxford University Press.

Price, H. (2005). Recent work on the arrow of radiation, contribution to this volume.

Wu, C.S., Ambler, E., Hayward, R.W., Hoppes, D.D. and Hudson, R.P. (1957). Experimental Test of Parity Conservation in Beta Decay. The Physical Review 105, 1413-1415.

Zeh, D. (2001). The Physical basis of the direction of time. 4th edition. Berlin: Springer-Verlag. 